\title{
Cooling of relativistic electron beams in chirped laser pulses
}

\author{
Samuel R. Yoffe ${ }^{a}$, Adam Noble $^{a}$, Yevgen Kravets ${ }^{b, a}$, and Dino A. Jaroszynski ${ }^{a}$ \\ ${ }^{a}$ Department of Physics, SUPA, University of Strathclyde, Glasgow G4 0NG, UK; \\ ${ }^{b}$ Centre de Physique Théorique, École Polytechnique, 91120, Palaiseau, France
}

\begin{abstract}
The next few years will see next-generation high-power laser facilities (such as the Extreme Light Infrastructure) become operational, for which it is important to understand how interaction with intense laser pulses affects the bulk properties of a relativistic electron beam. At such high field intensities, we expect both radiation reaction and quantum effects to play a significant role in the beam dynamics. The resulting reduction in relative energy spread (beam cooling) at the expense of mean beam energy predicted by classical theories of radiation reaction depends only on the energy of the laser pulse. Quantum effects suppress this cooling, with the dynamics additionally sensitive to the distribution of energy within the pulse. Since chirps occur in both the production of high-intensity pulses (CPA) and the propagation of pulses in media, the effect of using chirps to modify the pulse shape has been investigated using a semi-classical extension to the Landau-Lifshitz theory. Results indicate that even large chirps introduce a significantly smaller change to final state predictions than going from a classical to quantum model for radiation reaction, the nature of which can be intuitively understood.
\end{abstract}

Keywords: Radiation reaction, quantum effects, semi-classical model, beam cooling, chirped laser pulses

\section{INTRODUCTION}

In the coming years, a new generation of high-power laser facilities (such as the Extreme Light Infrastructure $(\mathrm{ELI})^{1}$ ) will become operational, delivering laser intensities well beyond those currently available. The extremely large fields generated by these pulses will allow qualitatively new physical regimes to be explored for the first time, in which both radiation reaction and quantum effects will have a dominant role to play.

An accelerating charge radiates energy (and momentum), and so must experience a recoil force. The emission must affect the dynamics of the particle. This is the concept of radiation reaction. Despite more than a century of investigation, radiation reaction remains a contentious area of physics. The most widely-accepted classical description of radiation reaction is based on (independent) attempts to include the effects of radiation emission by Lorentz $z^{2}$ and $A$ braham ${ }^{3}$ at the start of the twentieth century, which were later made fully relativistic by Dirac. ${ }^{4}$ The Lorentz-Abraham-Dirac (LAD) equation for a particle of mass $m$ and charge $q$ in an electromagnetic field $F$ reads

$$
\ddot{x}^{a}=\frac{f_{\mathrm{ext}}^{a}}{m}+\tau \Delta^{a}{ }_{b} \dddot{x}^{b}=-\frac{q}{m} F^{a}{ }_{b} \dot{x}^{b}+\tau\left(\dddot{x}^{a}-\ddot{x}_{b} \ddot{x}^{b} \dot{x}^{a}\right),
$$

where $f_{\text {ext }}^{a}=-q F^{a}{ }_{b} \dot{x}^{b}$ is the Lorentz force. Here, the constant $\tau:=q^{2} / 6 \pi m$ is the 'characteristic time' of the particle $\left(\simeq 6 \times 10^{-24} \mathrm{~s}\right.$ for an electron $)$. An overdot denotes differentiation with respect to proper time. Indices are raised and lowered with the metric tensor $\eta=\operatorname{diag}(-1,1,1,1)$, and repeated indices are summed from 0 to 3 . The $\dot{x}$-orthogonal projection $\Delta^{a}{ }_{b}:=\delta_{b}^{a}+\dot{x}^{a} \dot{x}_{b}$ removes any $\dot{x}$-component from the jerk term $\dddot{x}$, since $\Delta^{a}{ }_{b} \dot{x}^{b}=0$ due to the normalisation $\dot{x}^{a} \dot{x}_{a}=-1$. This ensures that $\ddot{x}$ is orthogonal to $\dot{x}$, such that the mass shell condition, $p^{a} p_{a}=-m^{2}$ where $p^{a}=m \dot{x}^{a}=(\gamma m, \vec{p})$, is preserved. Heaviside-Lorentz units are used with $c=1$.

Due to the presence of the third derivative $\dddot{x}$, the LAD equation exhibits 'runaway solutions'. These pathological solutions, which are highly unphysical and not observed, can be prevented at the cost of introducing a dependence on all future forces, known as preacceleration. Despite only forces within the small time $\tau$ having

Further author information:

S.R.Y.: E-mail: sam.yoffe@strath.ac.uk

D.A.J.: E-mail: d.a.jaroszynski@strath.ac.uk 
a significant influence, this response to all future forces that will act on the particle is not compatible with the concept of causality. The recent review ${ }^{5}$ describes these problems and proposed solutions in greater detail.

The moset widely-used alternative to the LAD description was introduced by Landau and Lifshitz. ${ }^{6}$ The radiation reaction (self-) force is treated as a perturbation about the Lorentz force,

$$
\ddot{x}^{a}=-\frac{q}{m} F_{b}^{a} \dot{x}^{b}+O(\tau) \quad \longrightarrow \quad \dddot{x}^{a}=-\frac{q}{m} \dot{x}^{c} \partial_{c} F_{b}^{a} \dot{x}^{b}-\frac{q}{m} F_{b}^{a} \ddot{x}^{b}+O(\tau),
$$

where once again the Lorentz force is substituted into the final term on the right-hand side. Collecting terms to leading order in $\tau$ we arrive at the Landau-Lifshitz equation:

$$
\ddot{x}^{a}=-\frac{q}{m} F^{a b} \dot{x}_{b}-\tau \frac{q}{m}\left(\partial_{c} F^{a b} \dot{x}_{b} \dot{x}^{c}-\frac{q}{m} \Delta^{a}{ }_{b} F^{b c} F_{c d} \dot{x}^{d}\right) .
$$

Note that $\Delta^{a}{ }_{c} F^{c b} \dot{x}_{b}=F^{a b} \dot{x}_{b}+\dot{x}^{a} F^{c b} \dot{x}_{c} \dot{x}_{b}=F^{a b} \dot{x}_{b}$.

This classical equation has found widespread use amongst the community. While there is mounting evidence to suggest that equation (3) is valid provided only that quantum effects can be ignored, ${ }^{7,8}$ the extreme conditions expected at ELI will take us into a regime where this is no longer possible. Instead, we must try to include some of the effects of quantum emission in order to make predictions for future experimental outcomes.

Quantum effects are typically considered to be negligible if the electric field observed by the particle $\hat{E}$ is much less than the Sauter-Schwinger critical field ${ }^{9,10}$ typical of QED processes, $E_{S}=1.32 \times 10^{18} \mathrm{~V} / \mathrm{m}$. That is, when the quantum nonlinearity parameter is small,

$$
\chi:=\frac{\hat{E}}{E_{S}} \ll 1
$$

Parameters obtainable at ELI are expected to approach $\chi \sim 0.8$, where quantum effects cannot be ignored. However, provided paramters are used such that $\chi^{2} \ll 1$ instead, a semi-classical modification to (3) should be valid. ${ }^{11}$

In order to model the weakly quantum regime, we briefy consider an important difference between classical and quantum emission. In the classical case, the particle radiates arbitrarily small amounts of energy at all frequencies. However, the quantum description requires the particle to emit entire photons of energy. The frequency of the emitted photons is therefore limited by the energy of the particle, which introduces a cutoff to the radiation spectrum. The continuous classical emission which underpins the classical descriptions of radiation reaction is therefore expected to overestimate the radiation produced as quantum effects become important. ${ }^{12}$ The Landau-Lifshitz equation is therefore extended to include this reduced emission, following Kirk, Bell and Arka, ${ }^{13}$ by scaling the radiation reaction force by a function of the quantum nonlinearity parameter $\chi$. The full expression for $g(\chi)$ involves a non-trivial integral over Bessel functions of the second kind. Instead, we use an approximation introduced by Thomas et al. ${ }^{14}$ based on fitting to numerical data,

$$
g(\chi)=\left(1+12 \chi+31 \chi^{2}+3.7 \chi^{3}\right)^{-4 / 9} .
$$

Predictions using this semi-classical model can be compared to those obtained from the classical Landau-Lifshitz theory in order to explore the role of quantum effects in the weakly quantum regime which will be available at ELI.

\section{COLLISION WITH INTENSE PLANE-WAVE LASER PULSES}

For a linearly polarised plane wave, the electromagnetic field tensor $F$ depends on spacetime only through the phase $\phi=\omega t-\vec{k} \cdot \vec{x}$, and takes the form

$$
\frac{q}{m} F_{b}^{a}=a(\phi)\left(\epsilon^{a} k_{b}-k^{a} \epsilon_{b}\right)
$$


where $k=(\omega, \vec{k})$ is the (null) propagation direction of the laser and $\epsilon$ the othogonal (transverse) polarisation vector. The function $a(\phi)$ is a dimensionless measure of the electric field strength. An $N$-cycle pulse modulated by a $\sin ^{2}$-envelope has been used, for which

$$
a(\phi)=\left\{\begin{array}{ll}
a_{0} \sin (\phi) \sin ^{2}(\pi \phi / L) & \text { for } 0<\phi<L \\
0 & \text { otherwise }
\end{array},\right.
$$

where $a_{0}$ is the dimensionless (peak) intensity parameter (or normalised vector potential) and $L=2 \pi N$ is the (total) pulse length (full-width half-maximum duration is $L / 2$ ). This pulse shape offers compact support, allowing the particles to begin and end in vacuum.

The total fluence (energy per unit area) of the pulse is proportional to

$$
\mathcal{E}=\int_{0}^{L} d \phi a^{2}(\phi)=\frac{3 \pi}{8} N a_{0}^{2} .
$$

In this work, $\mathcal{E}$ is kept constant, which fixes $a_{0}$ for each $N$. The classical Landau-Lifshitz prediction for the final-state particle distribution is completely determined by the fluence. ${ }^{15,16}$ By contrast, quantum effects are expected to depend directly on the intensity of the field itself, that is on the value of $a_{0}$. Maintaining constant fluence has the advantage that quantum effects can be studied while maintaining the same classical prediction.

Since our laser pulse is described by a plane wave, we consider the initial momenta to be strongly peaked around zero in the transverse directions ${ }^{16}$ and focus on the longitudinal properties of the distribution. The initial electron beam is then taken to be a Maxwellian distribution for the longitudinal momentum $p$ (in units of $m c$ ):

$$
f(\phi=0, p)=\frac{N_{P}}{\sqrt{2 \pi \theta}} \exp \left[-\frac{(p-\bar{p})^{2}}{2 \theta}\right]
$$

where $\theta$ is the variance of the distribution about its mean $\bar{p}$, and $N_{P}$ is the number of particles used to represent it. Instead of randomly sampling the distribution using a large number of particles, an iterative technique is used which facilitates efficient and accurate reconstruction of the distribution using far fewer particles. ${ }^{16}$ For the case of classical radiation reaction according to the Landau-Lifshitz equation, this method has been shown to be in excellent agreement with the analytical solution of the Vlasov equation. ${ }^{16,17}$

In order to describe the properties of the distribution, we introduce the relative momentum spread and the momentum skewness,

$$
\hat{\sigma}(\phi)=\frac{\sqrt{\theta(\phi)}}{\bar{p}(\phi)} \quad \text { and } \quad S(\phi)=\frac{\left\langle[p-\bar{p}(\phi)]^{3}\right\rangle}{\theta^{3 / 2}(\phi)},
$$

respectively. The former gives a measure of the beam quality, while the latter indicates how symmetric the distribution is about its mean.

We now consider the interaction of an energetic electron beam with a high-intensity laser pulse. Parameters have been chosen to be within the capabilities of ELI. An electron beam represented by $N_{P}=401$ particles with an initial momentum spread of $20 \%$ around $\sqrt{1+\bar{p}^{2}}=2 \times 10^{3}$ (which corresponds to an energy of approximately $1 \mathrm{GeV}$ ) is collided with pulses of length $N \in[5,200]$ cycles with constant $N a_{0}^{2}=9248$. For $N=20$ with a wavelength of $\lambda=800 \mathrm{~nm}$, this corresponds to a full-width half-maximum pulse duration of $27 \mathrm{fs}$ with peak intensity $2 \times 10^{21} \mathrm{~W} / \mathrm{cm}^{2}$.

Figure 1 presents a comparison of classical and semi-classical predictions for the cooling of a relativistic electron beam in the interaction with two pulse lengths, $N=10$ and $N=100$. For a wavelength of $\lambda=800 \mathrm{~nm}$, the former represents a pulse of total duration $27 \mathrm{fs}$ with peak intensity $I=3.96 \times 10^{21} \mathrm{~W} / \mathrm{cm}^{2}$, while the latter a total duration of $270 \mathrm{fs}$ and $I=3.96 \times 10^{20} \mathrm{~W} / \mathrm{cm}^{2}$. These intensities should be well within the capabilities at ELI without the need for extreme focussing. These unfocused pulses are better described by a plane wave.

Figure 1(a) shows the initial and final particle distribution for both pulse lengths predicted by both classical and semi-classical models. It should be noted that the classical final distributions sit directly on top of one 

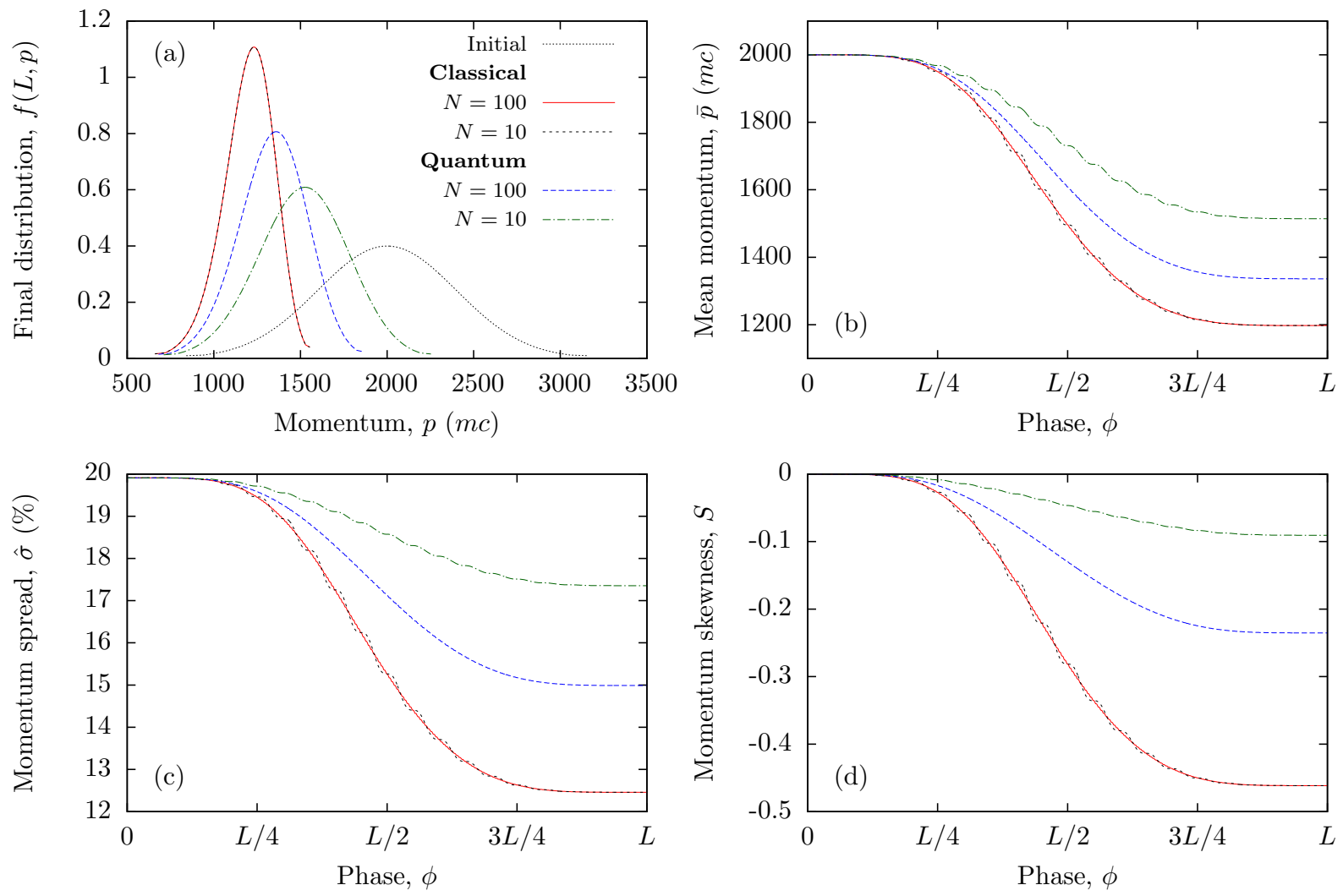

Figure 1. (Colour online) Comparison of classical and semi-classical predictions for the final-state properties of an electron beam colliding with pulses of $N=10,100$ cycles. Part (a): initial and final electron distributions. Classical predictions for both $N=10$ and 100 lie on top of one another. Parts (b)-(d): Evolution of the mean momentum $\bar{p}$, relative momentum spread $\hat{\sigma}$, and mometum skewness during the interaction. Classical results for the final distribution show beam cooling with a dependence on the total fluence only, while the quantum model predicts reduced cooling dependent on the pulse intesnsity.

another, supporting the statement that the final state classical predictions depend only on the total fluence of the laser, and not how the energy is distributed within it. The final distribution is significantly more sharply peaked around a much lower momentum than the initial distribution, demonstrating beam cooling. It also shows signs of antisymmetry, with a longer tail extending to lower momenta. In contrast, the semi-classical model predicts a different final distribution for each $N$. For $N=100$, the dimensionless intensity parameter $a_{0} \simeq 9.6$ and we see that quantum effects have become important. Beam cooling has been reduced, and this trend continues to $N=10$ (where $a_{0} \simeq 30$ ).

The fluctuation of the mean momentum as the beam passes through the pulse is shown in Fig. 1(b), with the relative momentum spread presented in Fig. 1(c). For the classical cases, we see that, despite the different pulse lengths behaving slightly differently during the evolution due to the different numbers of cycles, both converge on the same final state with $\bar{p}=1197.70$ and $\hat{\sigma}=12.46 \%$. For comparison, the analytical solution of the Vlasov equation with the Landau-Lifshitz theory for radiation reaction ${ }^{16}$ predicts $\bar{p}=1197.62$ and $\hat{\sigma}=12.53 \%$. Instead, the semi-classical model for $N=100$ predicts $\bar{p}=1336.28$ and $\hat{\sigma}=14.99 \%$. The results for $\hat{\sigma}$ show a reduction in beam cooling* ( $\hat{\sigma}$ does not reduce as much from the initial 20\%) with the beam remaining more energetic. For $N=10$, the semi-classical model predicts $\bar{p}=1514.40$ and $\hat{\sigma}=17.36 \%$, continuing the reduction of beam cooling. Note that, for $N=100$, we measure the average quantum nonlinear parameter $\langle\chi\rangle^{2}<\left\langle\chi^{2}\right\rangle<1 \times 10^{-4}$.

\footnotetext{
${ }^{*}$ Reduction in beam cooling means that there is less contraction of phase space than would be observed in the classical case. As such, the relative momentum spread remains larger than that predicted by the Landau-Lifshitz theory.
} 


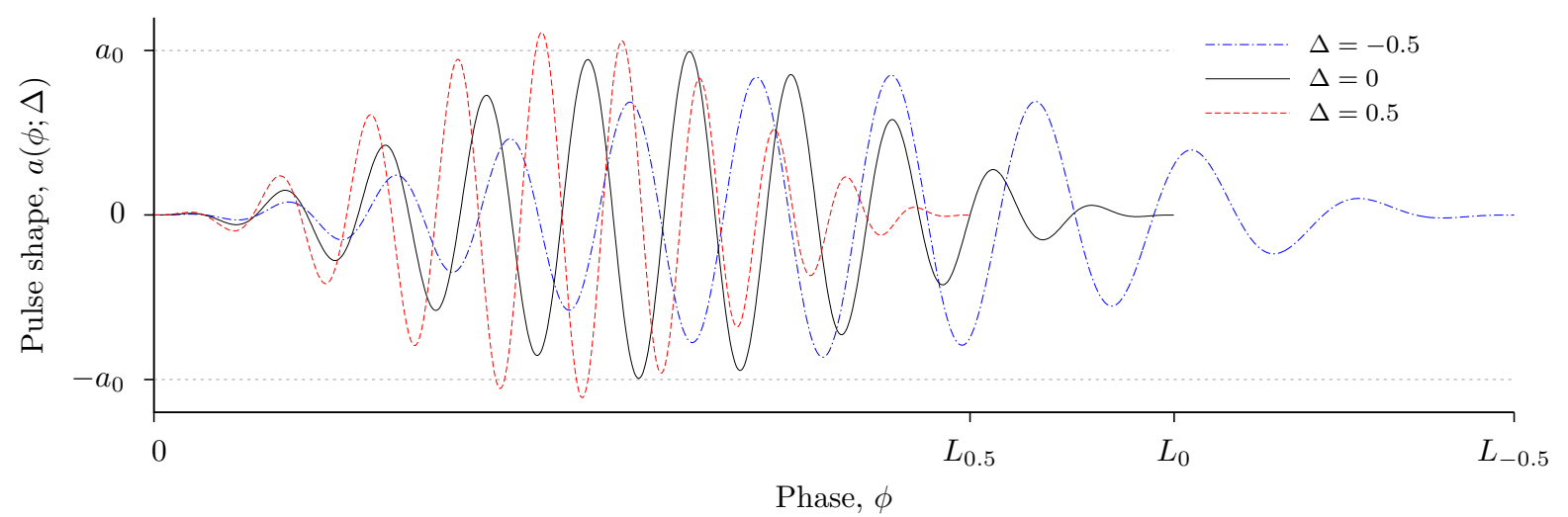

Figure 2. (Colour online) The dimensionless intensity profile for $N=10$ pulses with chirp rate $\Delta= \pm 0.5$, along with the original unchirped case. The pulse length $L_{\Delta}$ is given by equation (11).

For the more intense pulse with $N=10$, this increases to $\left\langle\chi^{2}\right\rangle<0.12$ and we expect the semi-classical model to remain a valid quantum approximation.

The momentum skewness of the distribution is given in Fig. 1(d), which shows the initially Gaussian $(S=0)$ profile becoming increasingly negatively skewed as the evolution proceeds. The analytical solution of the classical Vlasov equation ${ }^{16}$ predicts a final skewness of $S=-0.498$, while the numerical approach used here gives $S=$ $-0.462^{\dagger}$. This is reflected in Fig. 1(a) by the extended tail of the classical final distribution to lower momenta. This is a consequence of classical beam cooling, since it is the more energetic particles which are most influenced by radiation reaction. The semi-classical model suppresses radiation reaction more for precisely those particles, and as such the distribution becomes less negatively skewed.

\section{INTERACTION WITH CHIRPED PULSES}

The use of chirped-pulse amplification (CPA) to achieve high-intensity pulses makes their influence on beam dynamics directly relevant to future experiments. In addition, chirps naturally arise in the propagation of laser pulses in media. The results of the previous section indicate that, unlike the classical Landau-Lifshitz theory of radiation reaction, the semi-classical model is sensitive to how the energy is distributed in the pulse, not just the total energy. To investigate this further, we include a chirp in our plane wave model for the laser pulse.

We introduce the pulse length for an $N$-cycle chirped pulse

$$
L_{\Delta}=\frac{2 \pi N}{1+\Delta / 2}
$$

where $\Delta$ is the chirp rate. The linearly-chirped phase is then defined to be

$$
\eta(\phi ; \Delta)=\phi\left(1+\frac{\phi \Delta}{2 L_{\Delta}}\right) .
$$

If $\omega=\partial \phi / \partial t$ is our unchirped frequency, then we find

$$
\Omega(\phi ; \Delta)=\frac{\partial}{\partial t} \eta(\phi ; \Delta)=\omega\left(1+\frac{\phi \Delta}{L_{\Delta}}\right),
$$

indicating that the phase contains a linear chirp. Thus, at the end of the pulse $\phi=L_{\Delta}$ the frequency is $\Omega(L ; \Delta)=\omega(1+\Delta)$. (A positive chirp $\Delta>0$ therefore exhibits an increase in frequency as we move through the pulse.) The description of the pulse given in equation (7) is then replaced by

\footnotetext{
${ }^{\dagger}$ This numerical difference is due to the finite number of particles used to represent the distribution. ${ }^{16}$
} 

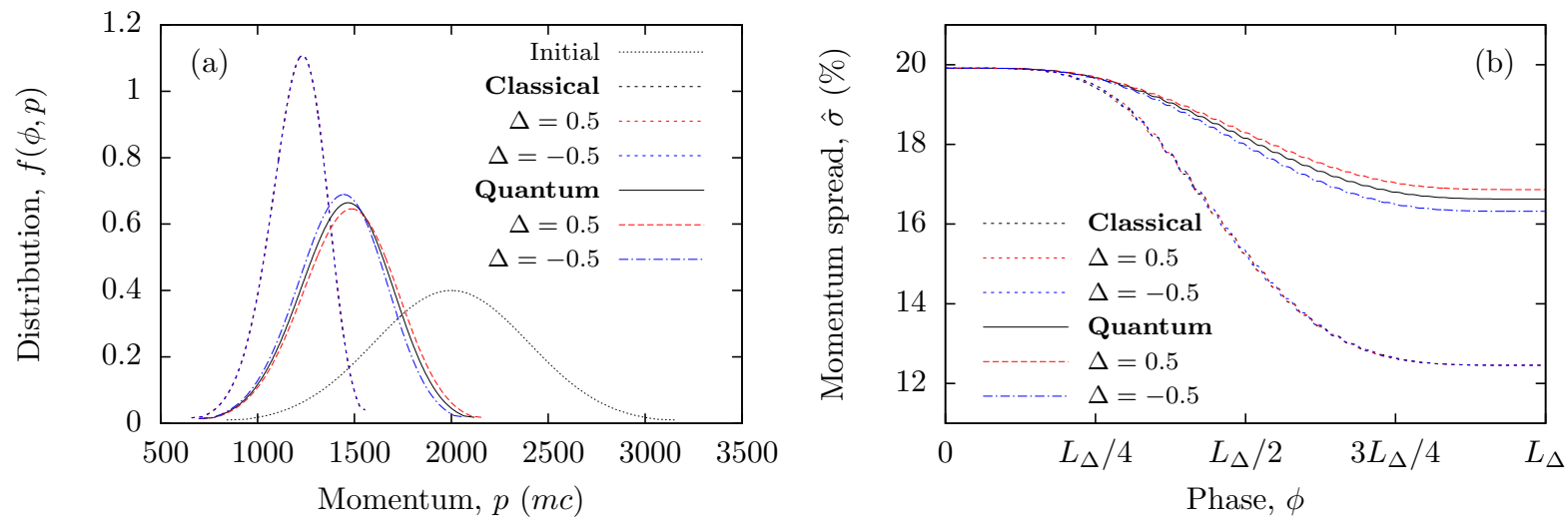

Figure 3. (Colour online) The influence of chirping in the semi-classical model of radiation reaction. Comparison of (a) initial and final distributions, and (b) evolution of the spread $\hat{\sigma}$, with and without a chirp. The classical final distribution and spread are insensitive to the chirp, despite a small variation during the evolution.

$$
a(\phi ; \Delta)=\left\{\begin{array}{ll}
a_{0} \sqrt{1+\frac{\Delta}{2}} \sin [\eta(\phi ; \Delta)] \sin ^{2}\left(\frac{\pi \phi}{L_{\Delta}}\right) & \text { for } 0<\phi<L_{\Delta} \\
0 & \text { otherwise }
\end{array} .\right.
$$

The electron beam encounters the same frequency at the front of the pulse for both positive and negative chirps, with the observed frequency then increasing (positive chirp) or decreasing (negative chirp) towards the rear of the pulse. The inclusion of the factor $\sqrt{1+\Delta / 2}=\sqrt{L_{0} / L_{\Delta}}$ in equation (14) ensures that pulses with the same $N$ and $a_{0}$ contain the same fluence, regardless of the chirp rate. This is important, as we want to investigate the role of the chirp itself. A comparison of pulses of different energy, not just different distribution of energy, would obscure this.

The pulse shape $a(\phi ; \Delta)$ is shown in Fig. 2 for $\Delta= \pm 0.5$, along with the unchirped pulse $(-)$. This illustrates how the increase in frequency caused by the positive chirp $(---)$ generates a shorter pulse length than the unchirped pulse. The amplitude is increased from $a_{0}$ (indicated by the horizontal dotted lines) such that the fluence remains constant. The negative chirp $(-\cdot-)$, on the other hand, causes an extended pulse of lower peak intensity for the same fluence.

Since the fluence is kept constant, predictions from the classical Landau-Lifshitz theory for the final properties of the beam are not affected by a chirp. This is confirmed in Fig. 3, where the influence of a chirp in the semiclassical model is also shown for the interaction of a $1 \mathrm{GeV}$ electron beam with $N=20\left(a_{0} \simeq 22\right)$ laser pulses. Figure 3(a) shows the initial and final distributions obtained using both the classical and semi-classical models, with and without chirps with $\Delta= \pm 0.5$. The evolution of the spread $\hat{\sigma}$ as the bunch travels through the pulse is shown in Fig. 3(b), which highlights the significant reduction in beam cooling when using the semi-classical model. Even with a relatively large chirp of $|\Delta|=0.5$, the impact of chirping the laser pulse can been seen to be a notably smaller effect than moving from the classical to the semi-classical model.

For the positively chirped case, a final spread $\hat{\sigma}_{+}=16.9 \%$ is found, compared to $\hat{\sigma}_{0}=16.6 \%$ for the unchirped pulse and $\hat{\sigma}_{-}=16.3 \%$ for the negative chirp. These changes in the spread are easily understood by considering the peak intensity encountered in each case. For the positive chirp, the pulse length is reduced because of the decrease in wavelength as we move through the pulse. To keep the fluence the same as for the unchirped pulse, the peak intensity must increase from $a_{0}$ to $a_{0} \sqrt{1+\Delta / 2}$. In turn, this increase leads to a higher instantaneous value of the quantum parameter, $\chi$, and thus increased suppression of the radiation reaction effect. Hence, beam cooling is further reduced by a positive chirp. In contrast, a negatively chirped pulse has a longer duration and a corresponding lower peak intensity, therefore quantum effects are less important and the radiation reaction term is less strongly suppressed. 


\section{CONCLUSIONS}

In the next few years, as new high-powered laser facilities come online, it is increasingly important to understand the physics that will occur at these unprecedented field strengths. Previously untested areas of physics will be experimentally probed for the first time, which requires a knowledge of fundamental principles such as radiation reaction in regimes for which quantum effects can no longer be ignored.

In this paper, a semi-classical extension to the Landau-Lifshitz theory of radiation reaction has been used to investigate the role of chirps in the interaction of relativistic electron beams with high-intensity laser pulses. Comparison is first made to classical predictions to identify the differences as some quantum effects are included in the model. Classical results are compared to analytical solution of the Vlasov equation, supporting the validity of the numerical technique used. A reduction in the amount of beam cooling is observed in the quantum case and found to depend on the intensity profile of the pulse itself, unlike classical predictions which only care about the total energy of the pulse. Chirps have been used to alter the distribution of energy within the pulses, with even a large chirp of $50 \%$ resulting in only a small modification to the final state properties, the origin of which is intuitively understood.

The results presented here are limited to the semi-classical case $\chi^{2} \ll 1$ due to the deterministic equation of motion used to describe radiation reaction. Strongly quantum regimes may be explored using similar techniques by adopting a stochastic equation where photon emission probabilities are determined by strong field QED, as in. ${ }^{18,19}$ This will be addressed in future work.

\section{ACKNOWLEDGMENTS}

This work is supported by the UK EPSRC (Grant EP/J018171/1); the ELI-NP Project; and the European Commission FP7 projects Laserlab-Europe (Grant 284464) and EuCARD-2 (Grant 312453). Datasets available online. ${ }^{20,21}$

\section{REFERENCES}

[1] http://www.eli-laser.eu/; http://www.eli-np.ro/.

[2] Lorentz, H. A., [The Theory of Electrons and its Applications to the Phenomena of Light and Radiant Heat], Stechert, New York (1916).

[3] Abraham, M., [The Classical Theory of Electricity and Magnetism], Blackie, London (1932).

[4] Dirac, P. A. M., "Classical Theory of Radiating Electrons," Proc. R. Soc. A 167, 148 (1938).

[5] Burton, D. A. and Noble, A., "Aspects of electromagnetic radiation reaction in strong fields," Contemporary Physics 55, 110-121 (2014).

[6] Landau, L. D. and Lifshitz, E. M., [The Classical Theory of Fields], Pergamon, London (1962).

[7] Kravets, Y., Noble, A., and Jaroszynski, D. A., "Radiation reaction effects on the interaction of an electron with an intense laser pulse," Phys. Rev. E 88, 011201(R) (2013).

[8] Spohn, H., "The critical manifold of the Lorentz-Dirac equation," Europhys. Lett. 50, 287 (2000).

[9] Sauter, F., "Über das Verhalten eines Elektrons im homogenen elektrischen Feld nach der relativistischen Theorie Diracs," Zeitschrift fr Physik 82, 742 (1931).

[10] Schwinger, J., "On Gauge Invariance and Vacuum Polarization," Phys. Rev. 82, 664 (1951).

[11] Erber, T., "High-energy electromagnetic conversion processes in intense magnetic fields," Rev. Mod. Phys. 38, 626 (1966).

[12] Ritus, V. I., "Quantum effects of the interaction of elementary particles with an intense electromagnetic field," J. Sov. Laser Res. 6, 497-617 (1985).

[13] Kirk, J. G., Bell, A. R., and Arka, I., "Pair production in counter-propagating laser beams," Plasma Phys. Control. Fusion 51, 085008 (2009).

[14] Thomas, A. G. R., Ridgers, C. P., Bulanov, S. S., Griffin, B. J., and Mangles, S. P. D., "Strong RadiationDamping Effects in a Gamma-Ray Source Generated by the Interaction of a High-Intensity Laser with a Wakefield-Accelerated Electron Beam," Phys. Rev. X 2, 041004 (2012). 
[15] Neitz, N. and Di Piazza, A., "Electron-beam dynamics in a strong laser field including quantum radiation reaction," Phys. Rev. A 90, 022102 (2014).

[16] Yoffe, S. R., Kravets, Y., Noble, A., and Jaroszynski, D. A., "Longitudinal and transverse cooling of relativistic electron beams in intense laser pulses," New Journal of Physics, Accepted (2015). arXiv:1504.03480.

[17] Noble, A., Burton, D. A., Gratus, J., and Jaroszynski, D. A., "A kinetic model of radiating electrons," J. Math. Phys. 54, 043101 (2013).

[18] Elkina, N. V., Fedotov, A. M., Kostyukov, I. Y., Legkov, M. V., Narozhny, N. B., Nerush, E. N., and Ruhl, H., "QED cascades induced by circularly polarized laser fields," Phys. Rev. ST Acc. Beams 14, 054401 (2011).

[19] Green, D. G. and Harvey, C. N., "Transverse Spreading of Electrons in High-Intensity Laser Fields," Phys. Rev. Lett. 112, 164801 (2014).

[20] Yoffe, S. R., Noble, A., Kravets, Y., and Jaroszynski, D. A., "Longitudinal beam cooling due to classical and semi-classical radiation reaction," (2015). http://dx.doi.org/10.15129/79f9c58d-7a43-4cc0-a613ebc028519e5b.

[21] Yoffe, S. R., Noble, A., Kravets, Y., and Jaroszynski, D. A., "Longitudinal beam cooling in chirped laser pulses," (2015). http://dx.doi.org/10.15129/44eaf2ee-f2a8-45b3-a05f-9575772d5ee6. 